\title{
The role of locus of control in nyaope addiction treatment
}

\author{
L Fernandes $^{a *}$ and KE Mokwena ${ }^{a}$ \\ aDepartment of Public Health, Sefako Makgatho Health Sciences University, Pretoria, South Africa \\ *Corresponding author, email: lucy_fernandes@embanet.com
}

\begin{abstract}
Full text available online at www.tandfonline.com/ojfp)
S Afr Fam Pract 2016; DOI:10.1080/20786190.2016.1223794

Background: Nyaope addiction is a significant health and social problem affecting some South African communities. With reported nyaope rehabilitation success rates of less than $3 \%$ and estimated dropout rates of $40 \%$, improvement in the rehabilitation rates is essential. As locus of control (LOC) is a recognised predictive factor in the onset of substance use and rehabilitation outcomes, a rehabilitation programme aligned to the LOC orientation of the user could result in improved recovery outcomes. This study aimed to determine the LOC of nyaope users.

Methods: Consenting adult male and female nyaope users voluntary participated in this quantitative study. After convenient sampling, 115 nyaope users admitted to drug rehabilitation centres registered and funded by the Gauteng Department of Social Development and 106 nyaope users from the streets of urban areas of Tshwane completed a self-administered validated questionnaire. LOC orientation was determined by giving participants 13 statements from which they had to choose the option that best described their situation in relation to drug addiction.

Results: A total of $24.5 \%$ (47/192) of the respondents could be classified as having an external LOC orientation while $75.5 \%$ $(145 / 192)$ were classified as having an internal LOC orientation.

Conclusions: By determining the LOC orientation of a nyaope user, rehabilitation programmes could be aligned to suit the personality of the user leading to a better chance of successful recovery with fewer cases of readmission.
\end{abstract}

Keywords: drug addiction, locus of control, nyaope, treatment

\section{Self-reported knowledge and use of emergency contraception among women presenting for termination of pregnancy}

\author{
O Osa-Izeko*, RD Govender and AJ Ross \\ Department of Family Medicine, University of KwaZulu-Natal, Durban, South Africa \\ ${ }^{*}$ Corresponding author, email: oroscope2000@yahoo.com
}

Abstract (Full text available online at www.tandfonline.com/ojfp)

S Afr Fam Pract 2016; DOI:10.1080/20786190.2016.1223797

Background: Emergency contraception (EC) is widely accepted as a safe method of preventing conception following unprotected coitus. Use of EC has been promoted in South Africa, yet uptake among women appears to remain low.

Aim: The aim of this study was to access knowledge and use of EC among women presenting for termination of pregnancy at a district hospital in KwaZulu-Natal.

Methods: This was a hospital-based cross-sectional study. Data were collected from women attending a termination of pregnancy (ToP) service at a district hospital using a questionnaire and analysed descriptively.

Results: A total of 218 women participated, of whom $25 \%$ were under 20 years of age and $87 \%$ were single. Reported knowledge of EC was good with $70 \%$ of participants indicating that they knew about EC. However, knowledge around EC was not complete, with less than $50 \%$ knowing the time interval between intercourse and $\mathrm{EC}$ action and some believing that EC acted by inducing an abortion. Reported usage of EC was only $40 \%$.

Conclusion: This study revealed that there is much more to be done to enhance knowledge and use of EC in this context. Reasons for the discrepancy between knowledge and usage need further exploration.

Keywords: emergency contraception, KwaZulu-Natal, pregnant women, self-reported knowledge, termination of pregnancy 